\title{
BOUNDED EXTENSION PROPERTY AND $p$-SETS
}

\author{
PER HAG
}

\begin{abstract}
The main result of this paper is a theorem which asserts that a closed subset of the compact Hausdorff space $X$ is a $p$-set for a uniform algebra $A$ on $X$ if and only if $S=\{f \in A ; \operatorname{Re} f>0\}$ has the so-called bounded extension property with respect to $F$. Similar results have been obtained by Bishop, Gamelin, Semadeni and the author.
\end{abstract}

1. Introduction. In the following $X$ is a compact Hausdorff space, $F$ is a closed subset of $X$ and $F^{\prime}$ denotes $X \backslash F$. A is a uniform algebra on $X$ and $S$ denotes a closed convex cone of $C(X)$, the space of all continuous complexvalued functions on $X$, equipped with the sup norm topology. $A \mid F$ and $S \mid F$ denote the set of all restrictions to $F$ of $A$ and $S$, respectively. For $\tilde{f} \in C(X)$, $f=\tilde{f} \mid F$ denotes the restriction of $\tilde{f}$ to $F$, and for $\mu \in M(X)$, the set of all complex Radon measures on $X, \mu_{F}$ denotes the restriction of $\mu$ to $F$. Likewise $\mu_{F^{\prime}}$ denotes the restriction of $\mu$ to $F^{\prime}$. By $A^{\perp}$ we understand the set of annihilating measures for $A$, and the dual cone $S^{*}$ of $S$ is the set

$$
S^{*}=\{\mu \in M(X) ; \operatorname{Re} \mu(\tilde{f})>0 \text { for all } f \in S\}
$$

[By $\operatorname{Re} \mu(\tilde{f})$ we understand $\operatorname{Re} \int_{X} \tilde{f} d \mu$.]

A closed subset $E$ of $X$ is a peak set for $A$ if there is a function $f \in A$ such that $f(x)=1$ for all $x \in E$ and $|f(y)|<1$ for all $y \in X \backslash E$. A $p$-set is the intersection of peak sets. In the following we restrict ourselves to proper $p$-sets, i.e. $E \neq X$.

The following definition is a generalization of a definition due to Michael and Pekczyński [7].

1.1. Definition. The closed convex cone $S$ is said to have the bounded extension property (BEP) w.r.t. $F$ if for each pair $\tilde{f} \in S, g \in S \mid F$ and every closed $G \subseteq X$ with $G \cap F=\varnothing$ and every $\epsilon>0$, there exists a $\tilde{g} \in S$ such that

(A) $\tilde{g} \mid F=g$,

(B) $\|\tilde{g}-\tilde{f}\|=\|g-f\|_{F}$,

(C) $\|\tilde{g}-\tilde{f}\|_{G}<\epsilon$.

(Here and in the following \|\| denotes the sup norm on $X$, while \|\|$_{F}$ denotes the sup norm on $F$, etc.)

Combining Theorem 2.2 and Lemma 4.2 of [6] we obtain immediately

Received by the editors July 31, 1978 and, in revised form, January 29, 1979.

AMS (MOS) subject classifications (1970). Primary 46J10. 
1.2. ThEOREM. With the same notation as above the following two conditions are equivalent:

(a) $S$ has the BEP w.r.t. F;

(b) $\nu \in S^{*} \Rightarrow \nu_{F} \in S^{*}$ and $\nu_{F^{\prime}} \in S^{*}$.

In the present paper we study the special case when

$$
S=\{f \in A ; \operatorname{Re} f(x)>0 \text { for all } x \in X\} .
$$

Theorem 2.1 asserts that $S$ has the BEP w.r.t. $F$ if and only if $F$ is a $p$-set for $A$. We also answer two questions raised in [6] using this result, although our earlier arguments could be used. The first result deals with elements in an arbitrary uniform algebra with nonnegative real parts, while the second result concerns the disc algebra.

2. The main result. The proof of the following theorem is the same as the proof of Proposition 4.3 of [6]. Since we are dealing with a more general situation, we give the argument for the sake of completeness.

2.1. Theorem. Let $X, F$ and $A$ be as given in the introduction and let $S$ be the closed convex cone given by

$$
S=\{f \in A ; \operatorname{Re} f(x)>0 \text { for all } x \in X\} .
$$

Then $S$ has the BEP w.r.t. $F$ if and only if $F$ is a p-set for $A$.

Proof. (i) Assume first that $S$ has the BEP w.r.t. $F$. Since $0,1 \in S$, it follows immediately from Definition 1.1 that $F$ is a $p$-set for $A$.

(ii) To prove the opposite implication, we need the following lemma.

2.2. Lemma. $S^{*}=M^{+}+A^{\perp}$, where $M^{+}$denotes the set of all nonnegative measures in $M(X)$.

Proof of Lemma 2.2. The inclusion $M^{+}+A^{\perp} \subseteq S^{*}$ is obvious. Suppose now that $f \in C(X)$ such that $\operatorname{Re} \mu(f) \geqslant 0$ for all $\mu \in M^{+}+A^{\perp}$. In particular, this holds for all $\mu \in A^{\perp}$. Hence $f \in A$. Since $\operatorname{Re} \mu(f)>0$ for all $\mu \in M^{+}$, it follows that $\operatorname{Re} f(x)>0$ for all $x \in X$. Consequently $f \in S$. By the separation theorem for convex sets it follows now that $M^{+}+A^{\perp}$ is $w^{*}$-dense in $S^{*}$. It is therefore sufficient to show that $M^{+}+A^{\perp}$ is $w^{*}$-closed. In this respect, let $\left\{\mu_{\alpha}+\nu_{\alpha}\right\}$ be a net in $M^{+}+A^{\perp}$ converging to $\sigma$ in the $w^{*}$-topology. Then we have

$$
\left\|\mu_{\alpha}\right\|=\int_{X} d \mu_{\alpha}=\int_{X} d\left(\mu_{\alpha}+\nu_{\alpha}\right) \rightarrow \int_{X} d \sigma .
$$

Hence $\left\{\mu_{\alpha}\right\}$ is a bounded net. Let $\mu$ be a $w^{*}$-cluster point of the elements of $\left\{\mu_{\alpha}\right\}$. Then $\mu \in M^{+}$and $\sigma-\mu$ is a $w^{*}$-cluster point of $\left\{\nu_{\alpha}\right\}$ and so in $A^{\perp}$. Therefore $\sigma \in M^{+}+A^{\perp}$.

Now assume that $F$ is a $p$-set of $A$. By the Glicksberg peak set theorem [4, Chapter II, Theorem 12.7], we know that $\mu_{F} \in A^{\perp}$ for all $\mu \in A^{\perp}$. It follows immediately that also $\mu_{F^{\prime}} \in A^{\perp}$ when $\mu \in A^{\perp}$. Let $\mu \in S^{*}$. By our lemma, 
$\mu=m+\nu$, where $m \in M^{+}$and $\nu \in A^{\perp}$. This implies

$$
\mu_{F}=m_{F}+\nu_{F} \in M^{+}+A^{\perp}
$$

and also

$$
\mu_{F^{\prime}}=m_{F^{\prime}}+\nu_{F^{\prime}} \in M^{+}+A^{\perp}
$$

since $m_{F}$ and $m_{F^{\prime}}$ are nonnegative measures and $\nu_{F}$ and $\nu_{F^{\prime}}$ are in $A^{\perp}$ by Glicksberg's theorem. Applying our lemma again, we obtain $\mu_{F}, \mu_{F^{\prime}} \in S^{*}$. From Theorem 1.2 we now conclude that $S$ has the BEP w.r.t. $F$.

REMARK. It is well known that in the case when $A$ is the disc algebra and $F \subseteq T$, the unit circle, then $F$ is a $p$-set for $A$ if and only if $F$ has one-dimensional Lebesgue measure 0 . Hence Theorem 2.1 can be considered as a generalization of Proposition 4.3 of [6]. Thus Theorem 2.1 answers the second question of $\$ 5$ of [6], affirmatively.

3. Consequences. In [6] the following result is proved.

3.1. Theorem. Let $X, F$ and $S$ be as in the introduction. Then the following conditions are equivalent

(a) $S$ has the BEP w.r.t. F.

(b) Given a pair $\tilde{f} \in S, g \in S \mid F$ and a lower semicontinuous function $\psi$ : $X \rightarrow(0, \infty]$ such that $|f(x)-g(x)|<\psi(x)$ for all $x \in F$, then there exists an extension $\tilde{g} \in S$ of $g$ such that $|\tilde{f}(x)-\tilde{g}(x)|<\psi(x)$ for all $x \in X$.

Combining Theorems 2.1 and 3.1 we obtain the following.

3.2. Theorem. With $X, F, A$ and $S$ as in Theorem 2.1., the following two conditions are equivalent

(a) $F$ is a p-set for $A$.

(b) Given a pair $\tilde{f} \in S, g \in S \mid F$ and a lower semicontinuous function $\psi$ : $X \rightarrow(0, \infty]$ such that $|f(x)-g(x)| \leqslant \psi(x)$ for all $x \in F$, then there exists an extension $\tilde{g} \in S$ of $g$ such that $|\tilde{f}(x)-\tilde{g}(x)|<\psi(x)$ for all $x \in X$.

If $X=\Delta$, the closed unit disc in the plane and $A$ is the disc algebra (considered as a uniform algebra on $\Delta$ ), we have the following consequence of Theorem 3.2.

3.3. Corollary. Let $F$ be a closed subset of the unit circle $T$ of one-dimensional Lebesgue measure 0 . Let $f$ be an arbitrary continuous function on $F$ with nonnegative real part. Assume also that $p: \Delta \rightarrow(0, \infty]$ is a lower semicontinuous function such that $|f(x)| \leqslant p(x)$ for all $x \in F$. Then there exists a function $\tilde{f} \in A$ such that

(i) $\tilde{f} \mid F=f$,

(ii) $|\tilde{f}(x)|<p(x)$ for all $x \in \Delta$,

(iii) $\operatorname{Re} \tilde{f}(x)>0$ for all $x \in \Delta$.

Proof. We observe that

$$
S \mid F=\{f \in C(F) ; \operatorname{Re} f(x)>0 \text { for all } x \in F\} .
$$


(For details, see [6]. The proof of the fact that $S \mid F$ is closed in $C(F)$ is in [1].) It is well known that $F$ is a proper $p$-set (or equivalently: a peak set) for the disc algebra $A$ if and only if $F$ is a closed subset of $T$ of one-dimensional Lebesgue measure 0 . Since $0 \in S$, the result follows immediately from our last theorem.

REMARK. Corollary 3.3 answers affirmatively the third question in $\$ 5$ of [6]. This result is also proved in [5] using a completely different technique. In fact, the result in [5] is slightly sharper than Corollary 3.3.

\section{BIBLIOGRAPHY}

1. E. L. Arenson, On the closure of the image of a convex set, Zap. Naučn. Sem. Leningrad. Otdel. Mat. Inst. Steklov (LOMI) 22 (1971), 171-174. (Russian) MR 45 \#871.

2. E. Bishop, A general Rudin-Carleson theorem, Proc. Amer. Math. Soc. 13 (1962), 140-143. MR 24 \# A3293.

3. T. W. Gamelin, Restriction of subspaces of $C(X)$, Trans. Amer. Math. Soc. 112 (1964), 278-286. MR 28 \# 5331.

4. __ Uniform algebras, Prentice-Hall, Englewood Cliffs, N. J., 1969.

5. J. Globevnik, Analytic extensions and selections (Preprint), University of Ljubljana, Yugoslavia, 1978.

6. P. Hag, Restrictions of convex subsets of $C(X)$, Trans. Amer. Math. Soc. 233 (1977), 283-294.

7. E. Michael and A. Polczyński, A linear extension theorem, Illinois J. Math. 11 (1967), 563-579. MR 36 \#671.

8. Z. Semadeni, Simultaneous extensions and projections in spaces of continuous functions, Lecture Notes, Univ. of Aarhus, 1965.

Department of MAthematics, University OF Trondhem, 7000 Trondhem, Norway (Current address)

Department of Mathematics, University of Michigan, AnN Arbor, Michigan 48109 